\title{
Efficiency enhancement in InGaN/GaN light-emitting diodes by decreasing the thickness of last barrier
}

\author{
Li-Wen Cheng ${ }^{1+}$, Yang Sheng ${ }^{2}$, Chang-Sheng $\mathrm{Xia}^{2}$, and Wei-Da $\mathrm{Hu}^{3}$ \\ ${ }^{1}$ College of Physics Science and Technology, Yangzhou University, Yangzhou 225002, China \\ ${ }^{2}$ Crosslight Software China, Suite 906,Building JieDi, 2790 Zhongshan Bei Road, Shanghai 200063, China \\ ${ }^{3}$ National laboratory for Infrared Physics, Shanghai Institute of Technical Physics, Chinese Academy of Science, Shanghai 200083,China
}

\begin{abstract}
InGaN/GaN light emitting diodes (LEDs) with different thickness of the last quantum barrier (LQB) near the $p$-layers is studied. Simulation results show that GaN-based LEDs with thinner LQB exhibit much higher output power and smaller efficiency droop than conventional LEDs due to the better electrons confinement in the quantum wells, which leads to efficient recombination of electrons and holes in the active region and thereby a significant enhancement in optical performance.
\end{abstract}

\section{INTRODUCTION}

GaN-based light emitting diodes (LEDs) have been vigorously developed and take the place of traditional incandescent and fluorescent lamps, due to its high brightness and low power consumption. However, the optical performance of GaN-based LEDs is suffering from a significant decrease, especially at high injection current, which of the exact origin is still under debate. Many possible mechanisms have been suggested to account for this efficiency droop situation, including polarization effect[1], auger nonradiative recombination, carrier overflow and poor hole injection efficiency[2]. To enhance the LED performance, numerous methods and novel designs have been proposed.

In this paper, LED structure with different thickness of the last quantum barrier(LQB) near the p-layers is theoretically studied by the APSYS software[3] which has been successfully applied to simulate plenty of realistic devices[4].Comparing LED with different thickness of LQB, electrons distribution in LEDs with thinner LQB is better, therefore, its performance is better.

\section{DEVICE STRUCTURE}

The conventional GaN-based LED used as a reference was grown on a c-plane sapphire substrate by metal-organic chemical vapor deposition (MOCVD) method. The epitaxial structure consists of a $25 \mathrm{~nm}$ thick low-temperature GaN nucleation layer, a 50-nm-thick undoped $\mathrm{GaN}$ layer, and a 3 - $\mu$ m-thick layer of n-type $\mathrm{GaN}$ (n-doping $=5 \times 10^{18} \mathrm{~cm}^{-3}$ ), followed by six periods of $\mathrm{In}_{0.15} \mathrm{Ga}_{0.85} \mathrm{~N} / \mathrm{GaN} \mathrm{MQWs}$, a $200-\AA$-thick layer of p-type $\left(\mathrm{p}\right.$-doping $\left.=2 \times 10^{18} \mathrm{~cm}^{-3}\right)$ $\mathrm{Al}_{0.15} \mathrm{Ga}_{0.85} \mathrm{~N}$ as electron blocking layer (EBL), and a $0.3-\mu \mathrm{m}$-thick layer of p-type $\mathrm{GaN}$ (p-doping $=2 \times 10^{18} \mathrm{~cm}^{-3}$ ) cap layer. The thicknesses of quantum well and $\mathrm{GaN}$ quantum

+ email: 1wcheng@yzu.edu.cn barrier are $2.5 \mathrm{~nm}$ and $15 \mathrm{~nm}$, respectively. Our studied structure was similar with the conventional LED except that the thickness of the last quantum barrier near the p-layers is decreased from $15 \mathrm{~nm}$ to $10 \mathrm{~nm}$ and $5 \mathrm{~nm}$, which is denoted as "structure A" and "structure B". The device geometry was designed with a rectangular shape of $300 \mu \mathrm{m} \times 300 \mu \mathrm{m}$.

\section{RESULTS AND DISCUSSIONS}

Energy band diagrams of the three LEDs at $25 \mathrm{~mA}$ are plotted in Fig.1. We can see that there is a severe band bending situation in the three LED structures due to polarization. For conventional LED, the energy band of the last quantum barrier is in the downward bending due to the electrostatic, so the effective barrier height of the EBL for the electrons is reduced. However, this situation has been alleviated in structure B and structure C (see the Fig 1(b) and Fig 1(c)) as the thickness of the LQB has been decreased. Especially, for the structure $\mathrm{C}(\mathrm{LQB}=5 \mathrm{~nm})$, the downwards band bending situation almost disappeared. Thus, the effective barrier height of the EBL for the electrons is increased from $281 \mathrm{meV}$ to $600 \mathrm{meV}$ as the thickness of the LQB is decreased from $15 \mathrm{~nm}$ to $5 \mathrm{~nm}$.

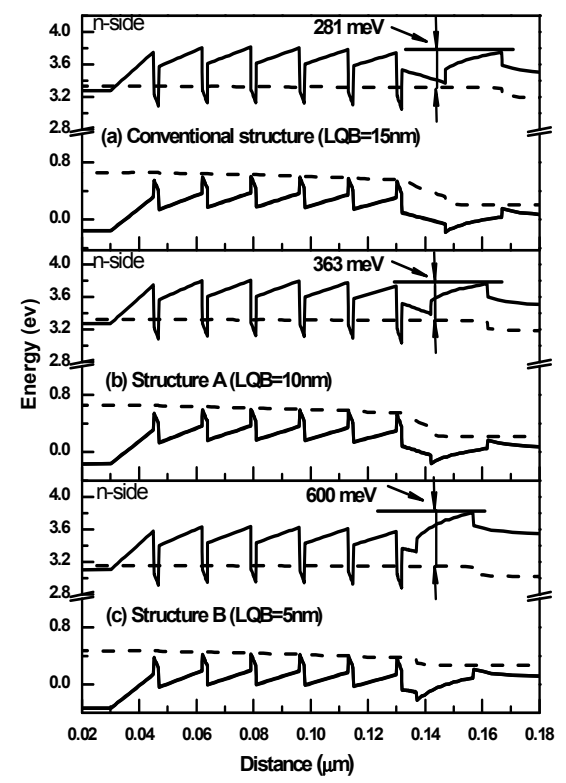

Fig.1. (a) Energy band diagram of the conventional structure,(b) structure A, and (c) structure $\mathrm{B}$ at $25 \mathrm{~mA}$

Figure 2 shows the electron concentration profiles of the three structures in the active region at $25 \mathrm{~mA}$. For conventional LED structure, it can be clearly seen that the 
electron concentrations are very high in the p-type GaN cap layer which indicates the large electron leakage. However, for the structure $\mathrm{B}$ and structure $\mathrm{C}$, the electron leakage is dramatically reduced. This situation coincides with the band diagrams analysis above. The main reason is that the effective barrier height of the EBL for the electrons is increased dramatically with the thickness of the LQB decreased.

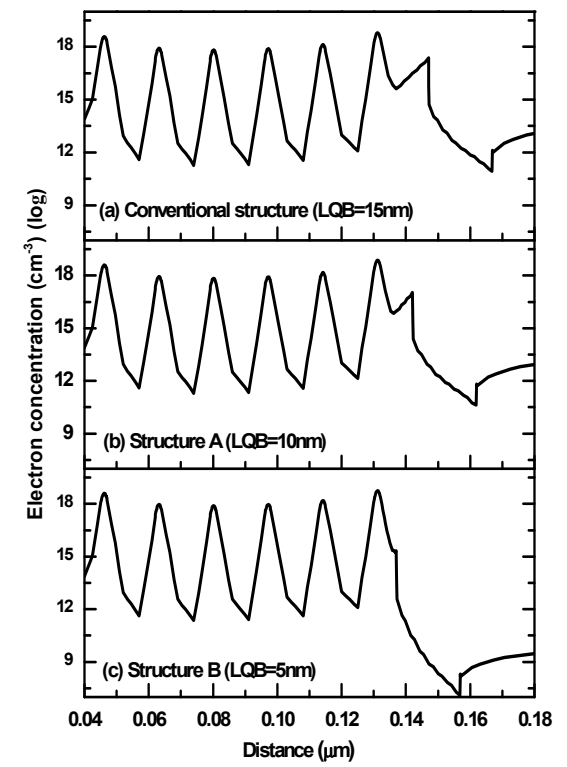

Fig.2. Electron concentrations of the three LED structures at $25 \mathrm{~mA}$

Figure 3 shows the vertical electron current density distributions near the active region of the three structures at $25 \mathrm{~mA}$. The electrons are injected from n-layers and then recombined with holes in quantum wells (QWs), which results in the decrease of electron current density along the distance. Fig. 3 shows that the electron leakage current which overflows from the active region to the p-layers of the conventional structure is quite serious. When the thickness of the LQB is decreased, the electron leakage current is markedly decreased.

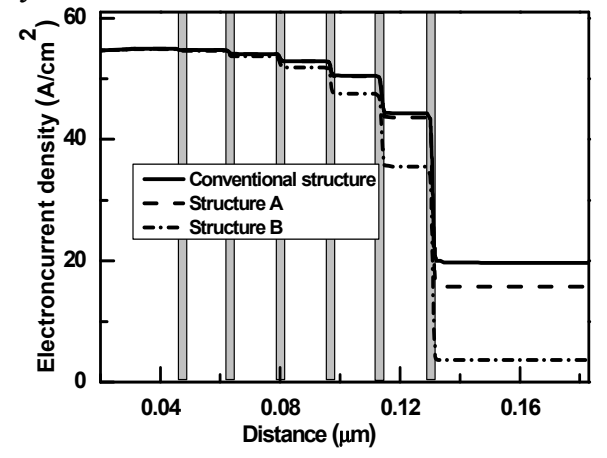

Fig.3. Electron current density distributions of the three LED structures at $25 \mathrm{~mA}$

The radiative recombination rate of the three structures at $25 \mathrm{~mA}$ is shown in Fig.4. Due to more electrons can be confined in the QWs with the thickness of the LQB decreased. It can be clearly seen that the radiative recombination rate is also increased.

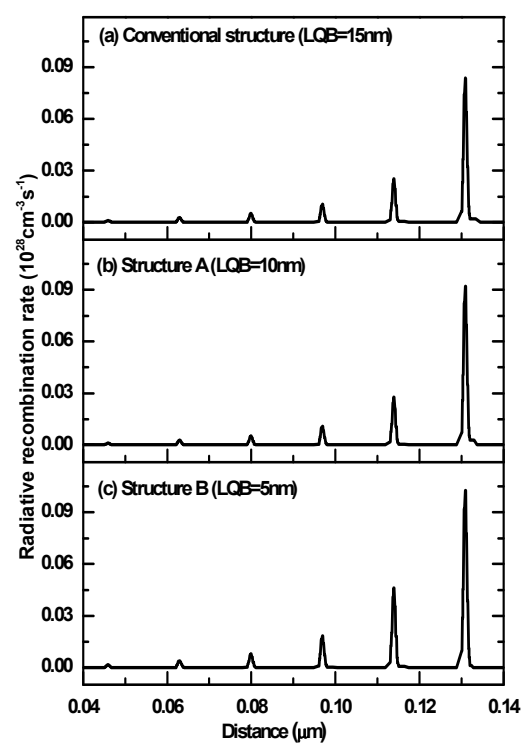

Fig.4. Radiative recombination rate of the three LED structures at $25 \mathrm{~mA}$

Because of the superior electron confinement in the QWs, the performance of the thinner thickness of the LQB LED is expected to be better than the conventional LED. Figure 5 shows the IQE and light output power as a function of current for the three LED structures under study, which indicates that the thinner thickness of LQB is, the better lighting efficiency is. It's worth noting here that the efficiency droop also be alleviated. If the droop ratio is defined as (Max_IQE-IQE at $25 \mathrm{~mA}$ )/Max_IQE, the droop ratio of the conventional LED is about $36.2 \%$. However, the efficiency droop situation is improved and the droop ratio be reduced to $16.8 \%$.

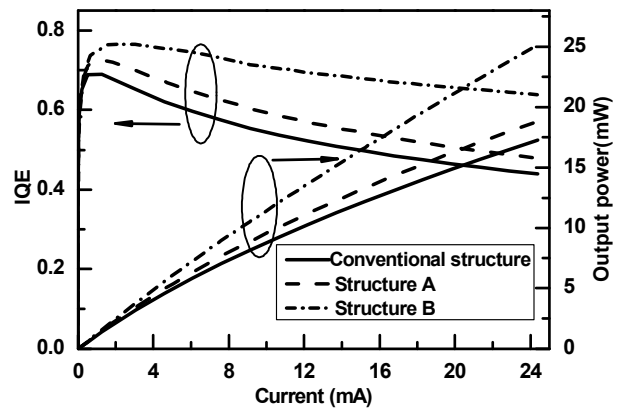

Fig. 5. IQE and light output power as a function of current for the three LED structures under study.

\section{CONCLUSIONS}

In this paper, simulation work is made to compare the different thickness of the last quantum barrier LEDS. Simulation results show that the thinner thickness of the LQB LED made electron confinement in the quantum wells more effective. Its significant advantage may help design LEDs with better performance.

\section{REFERENCES}

[1] M.H. Kim, M. F. Schubert, Q. Dai, et al, Appl. Phys. Lett. 91,183507 (2007)

[2] H. Han, D. Y. Lee, S. J. Lee, et al. Appl. Phys. Lett. 94, 231123(2009).

[3] APSYS User's Manual 2013, http://www.crosslight.com

[4] C.S. Xia, Z.M. Simon Li, Z.Q. Li, and Y.Sheng. Appl. Phys. Lett. 102, 013507 (2013) 